$(p=0.192)$. Disease progression and overall survival did not differ between groups $(p=0.537, p=0.465$, respectively). However, patients operated with MIS had almost 3 times risk to recur at their vaginal cuff or pelvis (Odds Ratio (OR) 95\% Confidence Interval (CI) 2.80 (1.80-4.36)). In a multivariable analysis, including age, comorbidities, disease stage, CA-125 and lymph-vascular space invasion, MIS was associated with an increased risk for local (vaginal cuff or pelvic) recurrence (OR 95\% CI 3.30 (1.69-6.48)).

Conclusions In women with HGEC, MIS was associated with higher rates of local recurrence as compared to laparotomy

\section{OP013/\#417 COST-EFFECTIVENESS OF DOSTARLIMAB IN ADVANCED RECURRENT DEFICIENT MISMATCH REPAIR ENDOMETRIAL CANCER PATIENTS}

${ }^{1} S$ Dioun*, ${ }^{2} \mathrm{~L}$ Chen, ${ }^{2} \mathrm{~A}$ Gockley, ${ }^{3} \mathrm{~A}$ Melamed, ${ }^{2} \mathrm{C}$ St Clair, ${ }^{3} \mathrm{~A}$ Tergas, ${ }^{3} \mathrm{~J}$ Hou, ${ }^{2} \mathrm{~F}$ Collado, 2J Wright. 'Columbia University Medical Center, Obstetrics and Gynecology, New York, USA; ${ }^{2}$ Columbia University College of Physicians and Surgeons, Gynecologic Oncology, New York City, USA; ${ }^{3}$ Columbia University, Gynecologic Oncology, New York, USA

\subsection{6/ijgc-2021-IGCS.30}

Objectives Women with recurrent endometrial cancer who fail carboplatin and paclitaxel have a poor prognosis with few effective options. The recent GARNET Trial showed promising results for dostarlimab in these patients. We developed a decision model to compare the cost-effectiveness of dostarlimab to other treatment options in patients with progressive/recurrent deficient mismatch repair (dMMR) endometrial cancer who have failed first-line chemotherapy.

Methods A Markov model was created to simulate the clinical trajectory of women with progressive/recurrent dMMR endometrial cancer who failed carboplatin and paclitaxel (figure 1). The initial decision point in the model was treatment with either dostarlimab, pembrolizumab or pegylated liposomal doxorubicin (PLD). Model probabilities, cost and utility values were derived with assumptions drawn from published literature. The effectiveness was measured in terms of quality adjusted life years (QALYs) gained. The primary outcome was incremental cost-effectiveness ratios (ICERs), expressed in 2018 US dollars/QALYs. One-way sensitivity analyses were performed to vary the assumptions across a range of plausible values.

Results PLD was the least costly strategy at $\$ 54,307$, followed by pembrolizumab $(\$ 160,780)$ and dostarlimab (\$251,132). PLD was cost-effective compared with
Abstract OP013/\#417 Table 1 Outcomes of study population

\begin{tabular}{|l|c|c|c|c|c|}
\hline \multicolumn{1}{|c|}{ Treatment } & $\mathrm{N}$ & Response Rate (\%) & Total Costs (\$) & QALYs & ICER \\
\hline PLD & 2,000 & 10 & $108,615,907$ & 1,102 & N/A \\
\hline Pembrolizumab & 2,000 & 29 & $321,561,768$ & 1,876 & Extended dominance \\
\hline Dostarlimab & 2,000 & 42 & $502,265,891$ & 3,074 & 199,621 \\
\hline
\end{tabular}

Assuming a population of 6,000 progressive/recurrent dMMR endometrial cancer patients

dostarlimab with an ICER of $\$ 199,621$, while pembrolizumab was subjected to extended dominance (table 1). Multiple one-way sensitivity analyses did not substantially impact the cost-effectiveness.

Conclusions Dostarlimab is associated with greater survival compared with other treatments for women with recurrent dMMR endometrial cancer. However, the agent is substantially more costly.

\section{OP014/\#480 TARGETED MOLECULAR TESTING IN ENDOMETRIAL CARCINOMA: VALIDATION OF A RESTRICTED TESTING PROTOCOL}

${ }^{1} \mathrm{~A}$ Talhouk ${ }^{*},{ }^{1} \mathrm{~A}$ Jamieson, ${ }^{2} \mathrm{E}$ Crosbie, ${ }^{3} \mathrm{~A}$ Taylor, ${ }^{4} \mathrm{D}$ Chiu, ${ }^{5} \mathrm{~S}$ Leung, ${ }^{6} \mathrm{M}$ Grube, ${ }^{6} \mathrm{~S}$ Kommoss, ${ }^{7} \mathrm{C}$ Gilks, ${ }^{1} \mathrm{~J}$ Mcalpine, ${ }^{8} \mathrm{~N}$ Singh. ${ }^{1}$ University of British Columbia, Gynecologic Oncology, Vancouver, Canada; ${ }^{2}$ University of Manchester, Division of Cancer Sciences, School of Medical Sciences, Manchester, UK; ${ }^{3}$ Royal Marsden Hospital, Gynaecology Unit, London, UK; ${ }^{4}$ University of British Columbia, Pathology and Laboratory Medicine, Vancouver, Canada; ${ }^{5}$ Molecular Oncology, University of British Columbia, Vancouver, Canada; ${ }^{6}$ Tuebingen Women's Hospital, Gynecologic Oncology, Tuebingen, Germany; ${ }^{7} U B C$, Vancouver General Hospital, Pathology and Laboratory Medicine, Vancouver, Canada; ${ }^{8}$ Barts Health NHS Trust, Cellular Pathology, London, UK

\subsection{6/ijgc-2021-IGCS.31}

Objectives The World Health Organization (WHO) endorsed molecular classification of endometrial carcinoma (EC) to be incorporated in routine diagnostic workup, by evaluating p53 and mismatch repair (MMR) protein immunohistochemistry (IHC), as well as pathogenic mutations in the gene encoding DNA polymerase epsilon (POLE). The latter is currently the least affordable or accessible step. We investigated whether POLE testing can be omitted in patients who based on stage, grade and lymphovascular space invasion (LVI) criteria would not usually be directed to adjuvant therapy.

Methods Using data from a single cancer centre $(n=460)$ in Vancouver, and a population-based cohort in Tubingen $(n=452)$, we compared the WHO recommended molecular testing of the entire cohort $(n=912)$ with a restricted protocol: p53 and MMR IHC on all cases, but POLE sequencing

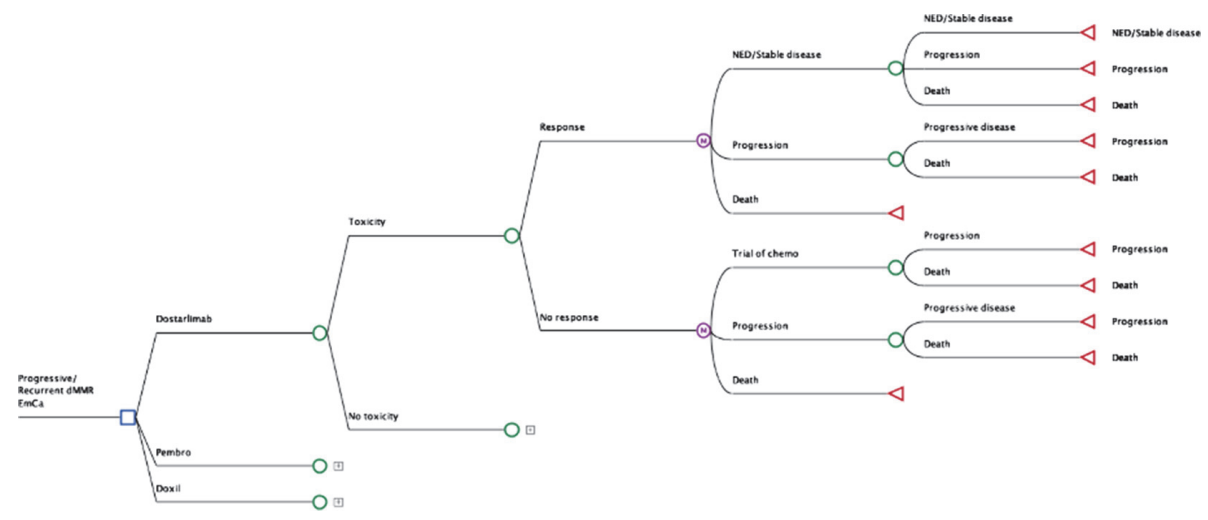


not done on 'very low-risk' ECs, defined as Stage 1A, G1/ G2, no LVI, MMR proficient and without p53 abnornalities.

Results $30 \%$ of full cohort and 38\% of population-based patients were classified as 'very low-risk', and did not undergo POLE testing. 'Very low-risk' ECs with unknown POLE status showed excellent clinical outcomes in both univariable and multivariable survival models. Amongst G1/G2 EEC, 14/566 (2.5\%) were p53abn, and G1/G2 EEC constituted 14/166 (8.4\%) of all p53abn ECs.

Conclusions Molecular classification of EC can be safely and more pragmatically incorporated into routine clinical practice using universal MMR and p53 IHC, and foregoing POLE testing in 'very low-risk' ECs where this has no therapeutic impact. Restricting molecular testing to high-grade/high-risk EC would miss some p53abn patients.

\section{OP015/\#492 FURTHER STRATIFICATION OF NO SPECIFIC MOLECULAR PROFILE (NSMP/P53WT) ENDOMETRIAL CARCINOMAS TO REFINE PROGNOSIS AND IDENTIFY THERAPEUTIC OPPORTUNITIES}

\begin{abstract}
${ }^{1} E$ Thompson, ${ }^{2}$ J Huvila, ${ }^{3} \mathrm{D}$ Chiu, ${ }^{4} \mathrm{~S}$ Leung, ${ }^{3} \mathrm{~A}$ Lum, ${ }^{5} \mathrm{~A}$ Jamieson, ${ }^{6} \mathrm{M}$ Köbel, ${ }^{7} \mathrm{M}$ Plante, ${ }^{8} \mathrm{~S} S$ cott, ${ }^{9} \mathrm{~S}$ Salvador, ${ }^{10} \mathrm{D}$ Vicus, ${ }^{11} \mathrm{~L}$ Helpman, ${ }^{12} \mathrm{M}$ Kinloch, ${ }^{13} \mathrm{~K}$ Grondin, ${ }^{14} \mathrm{~J}$ Irving, ${ }^{15} \mathrm{~A}$ Talhouk, ${ }^{3} \mathrm{D}$ Huntsman, ${ }^{16} \mathrm{~S}$ Kommoss, ${ }^{17} \mathrm{C}$ Gilks, ${ }^{5} \mathrm{~J}$ Mcalpine. ${ }^{1}$ Univeristy of British Columbia, Molecular Oncology, Vancouver, Canada; ' University of Turku, Biomedicine, Turku, Finland; ${ }^{3}$ Molecular Oncology, University of British Columbia, Vancouver, Canada; ${ }^{4}$ University of British Columbia, Genetic Pathology Evaluation Centre, Vancouver, Canada; ${ }^{5}$ University of British Columbia, Gynecologic Oncology, Vancouver, Canada; ${ }^{6}$ University of Calgary, Department of Pathology and Laboratory Medicine, Calgary, Canada; ${ }^{7}$ Hotel Dieu de Quebec, Gynecology Oncology, Quebec, Canada; ${ }^{8}$ Dalhousie University, Obstetrics and Gynaecology, Halifax, Canada; ${ }^{9}$ McGill University, Jewish General Hospital, Gynecology Oncology, Montreal, Canada; ${ }^{10}$ Sunnybrook Health Sciences Centre, Gynecologic Oncology, Toronto, Canada; ${ }^{11}$ McMaster University, Juravinski Cancer Center, Hamilton Health Sciences, Gynecologic Oncology, Hamilton, Canada; ${ }^{12}$ University of Saskatchewan, Pathology and Laboratory Medicine, Saskatoon, Canada; ${ }^{13}$ Université Laval, CHU de Québec, Pathology, Quebec City, Canada; ${ }^{14}$ University of British Columbia, Royal Jubilee Hospital, Pathology and Laboratory Medicine, Victoria, Canada; ${ }^{15}$ University of British Columbia, Obstetrics and Gynecology, Vancouver, Canada; ${ }^{16}$ Tuebingen Women's Hospital, Gynecologic Oncology, Tuebingen, Germany; ${ }^{17}$ UBC, Vancouver General Hospital, Pathology and Laboratory Medicine, Vancouver, Canada
\end{abstract}

\subsection{6/ijgc-2021-IGCS.32}

Objectives Molecular classification identifies $>50 \%$ of endometrial cancers (ECs) as having 'no specific molecular profile/ NSMP'; without mismatch repair deficiency, p53 IHC abnormalities, or pathogenic POLE mutations. Clinical presentation and outcomes within NSMP ECs are diverse and optimal treatment unclear with new ESMO/ESTRO/ESP guidelines unchanged for this molecular subtype. Better biomarkers are needed to predict if and what adjuvant therapies are needed. Methods We characterized the clinicopathological and molecular (IHC+NGS) profiles of 1047 NSMP ECs in women from population-based and institutional cohorts, testing for associations with treatment response and outcomes.

Results Key pathologic and molecular features associated with survival parameters $(\mathrm{p}<0.01)$ are tabulated below. $31 \%$ of NSMP ECs had CTNNB1 mutations, however, associations with outcomes (PFS) were observed only within Gr1/2 earlystage endometrioid $\operatorname{ECs}(\mathrm{p}=0.03)$, or if restricted to ECs without substantial LVI or L1CAM overexpression $(\mathrm{p}<0.005)$. TP53 mutations (with normal p53IHC) were discovered in 41 women with a trend $(p=0.06)$ to worse survival. On

\begin{tabular}{|c|c|c|c|c|}
\hline & Full cohort & Stage IA & Stage IB & St IIIIV \\
\hline Total & $1047(100 \%)$ & $673(67 \%)$ & $164(16 \%)$ & $\begin{array}{ll}175(17 \%) \\
\end{array}$ \\
\hline Age -median (range) & $61(22-96)$ & $59(22-94)$ & $66(32-93)$ & 63(32-96) \\
\hline BMI-median (range) & 31 (16-82) & $31(16-82)$ & $31(16-66)$ & $29(16-53)$ \\
\hline Grade 1/2/3 (\%) & $69 / 17 / 14 \%$ & 79/15/7\% & $66 / 19 / 15 \%$ & $34 / 29 / 37 \%$ \\
\hline Histof endometrioid & 974 (93\%) & 641 (96\%) & $159(97 \%)$ & $142(82 \%)$ \\
\hline $\begin{array}{c}\text { LVI } \\
\text { neg/focal/substantial }\end{array}$ & $82 / 15 / 3 \%$ & $93 / 6 / 1 \%$ & $73 / 20 / 6 \%$ & $45 / 45 / 10$ \\
\hline ESMO low/int/HIR/high & \begin{tabular}{|l}
$52 / 10 / 14 / 2$ \\
3
\end{tabular} & 78/0/17/5 & $0 / 68 / 17 / 15$ & 0/0/0/94 \\
\hline ER neg/weak/strong & 7/16/77 & 5/14/81 & $7 / 19 / 73$ & $17 / 24 / 59$ \\
\hline PR neg/weak/strong & $11 / 20 / 69$ & $8 / 18 / 74$ & $5 / 29 / 66$ & $31 / 19 / 50$ \\
\hline LICAM overexpression & $15 \%$ & $13 \%$ & $13 \%$ & $24 \%$ \\
\hline ARID1A loss (IHC) & $33 \%$ & $31 \%$ & $38 \%$ & $36 \%$ \\
\hline PIK3CA mutation & $39 \%$ & $39 \%$ & $32 \%$ & $35 \%$ \\
\hline $\begin{array}{c}\text { Post-surgical Rx (\%) } \\
\text { none/VB/EBRT/chemo+/-RT }\end{array}$ & $65 / 14 / 9 / 12$ & $83 / 11 / 4 / 2$ & $38 / 39 / 14 / 9$ & $21 / 5 / 25 / 47$ \\
\hline Overall survival event & $198(19.5 \%)$ & $79(12 \%)$ & $38(24 \%)$ & $64(37 \%)$ \\
\hline $\begin{array}{l}\text { Disease specific survival } \\
\text { event }\end{array}$ & $82(8 \%)$ & $17(3 \%)$ & $11(7 \%)$ & $43(26 \%)$ \\
\hline $\begin{array}{l}\text { Progression/recurrence } \\
\text { events }\end{array}$ & $102(10.4 \%)$ & 37 (5.7\%) & $19(12 \%)$ & $44(29 \%)$ \\
\hline
\end{tabular}

Cox regression and Kaplan-meier analysis demonstrate statistically significant associations w/outcomes (OS, DSS, PFS) $(\mathrm{p}<0.01)$ for all parameters except Age, BMI, and PIK3CA (OS +DSS only) + ARID1A (OS only)

multivariable analysis only grade (3vs.1/2) maintained significance. $8 \%$ of this cohort would be eligible for current molecular classification de-escalation trials. Treatment received did not impact survival within low-, intermediate-, or high-intermediate risk NSMP ECs. Within high-risk, the most favorable outcomes were observed in women who received pelvic radiation with no observed benefit of chemotherapy.

Conclusions Additional prognostic stratification of NSMP ECs can be achieved with both pathologic and molecular features. Further study within NSMP subgroups may identify conventional, hormonal or targeted therapies that are more effective.

\section{OP016/\#563 MORBIDITY AND QUALITY OF LIFE OF SENTINEL LYMPH NODE MAPPING IN ENDOMETRIAL CANCER. INTERIM ANALYSIS OF A PROSPECTIVE RANDOMIZED TRIAL (ALICE TRIAL)}

${ }^{1} B$ Goncalves*, 'L Kumagai, ${ }^{1} \mathrm{H}$ Mantoan, ${ }^{1} \mathrm{C}$ Faloppa, ${ }^{2} \mathrm{R}$ Ribeiro, ${ }^{3} \mathrm{R}$ Moretti-Marques, ${ }^{4} \mathrm{C}$ andrade, ${ }^{5} \mathrm{~A}$ Lopes, ${ }^{1} \mathrm{~L}$ Badiglian-Filho, ${ }^{1} \mathrm{~A}$ Menezes, ${ }^{6} \mathrm{M}$ Chen, ${ }^{7} \mathrm{~A}$ Guimaraes, ${ }^{1} G$ Baiocchi. 'AC Camargo Cancer Center, Gynecologic Oncology, Sao Paulo, Brazil: ${ }^{2}$ Hospital Erasto Gaertner, Surgical Oncology, Curitiba, Brazil; ${ }^{3}$ Albert Einstein Hospital, Gynecologic Oncology, São Paulo, Brazil; ${ }^{4}$ Hospital de Amor, Gynecologic Oncology Department, Barretos, Brazil; ${ }^{5}$ Brazilian Institute for Cancer Control, Gynecologic Oncology, São Paulo, Brazil; ${ }^{6}$ AC Camargo Cancer Center, Radiation Oncology, São Paulo, Brazil; ${ }^{7}$ AC Camargo Cancer Center, Medical Oncology, São Paulo, Brazil

\subsection{6/ijgc-2021-IGCS.33}

Objectives Despite the growing evidence of sentinel lymph node mapping (SLN) in endometrial cancer, studies addressing morbidity and impact on quality of life (QoL) are still scarce. Our aim was to evaluate treatment morbidity and QoL for SLN \pm systematic lymph node dissection (LND) in endometrial cancer. 